\title{
La dimensión transmedia de Harry Potter: rasgos de las extensiones canónicas
}

\section{The transmedia dimension of Harry Potter: features of the canonical extensions}

\author{
María Melle Goyanes \\ Graduada en Periodismo \\ (Facultad de Ciencias de la Comunicación de Santiago de Compostela) \\ Alicia Pardo Crego \\ Graduada en Periodismo \\ (Facultad de Ciencias de la Comunicación de Santiago de Compostela)

\section{Carlos Toural Bran} \\ Periodista y profesor de periodismo \\ (Facultad de Ciencias de la Comunicación de Santiago de Compostela)

\section{Xosé López García} \\ Periodista y catedrático de periodismo \\ (Facultad de Ciencias de la Comunicación de Santiago de Compostela)
}

Fecha de recepción: 28 de diciembre de 2016

Fecha de revisión: 20 de marzo de 2017

Fecha de publicación: 1 de julio de 2017

Para citar este artículo: Melle Goyanes, M., Pardo Crego, A., Toural Bran, C. y López García, X. (2017): La dimensión transmedia de Harry Potter: rasgos de las extensiones canónicas, Icono 14, volumen 15 (2), pp. 1-24. doi: 10.7195/ri14.v15i2.1023 


\section{Resumen}

La digitalización de la sociedad ha provocado el surgimiento de las narrativas transmedia, una nueva forma de contar historias a través de múltiples plataformas. El presente artículo profundiza en este concepto aplicado a un caso práctico, el de Harry Potter, en el que se estudian sus diversas extensiones en base a su medio, su audiencia y su función. La metodología se ha basado en la revisión bibliográfica, las entrevistas a fuentes, y el análisis individualizado de cada una de las extensiones. Como resultado, se ha alcanzado una concepción propia de transmedia que ha hecho posible delimitar y establecer las fases por las que ha pasado este tipo de narrativas en Potter: desde el boom de los inicios y el estancamiento posterior, hasta la nueva era que acaba de comenzar con el lanzamiento de la nueva saga de películas, y que promete repetir el éxito de los años anteriores.

Palabras clave: Transmedia - Storytelling - Comunicación digital - Plataformas Harry Potter - Adaptaciones - Consumidores

\section{Abstract}

The digitalisation of society has incurred into the raised of the transmedia storytelling, a new way of telling stories across multiple platforms. The present review go deep into this concept applied to a practical case, Harry Potter's, in which is studied their different extensions in terms of its medium, its audience and its function. The methodology has been based on the bibliographic review, interviews with sources and an individual analysis of each platform. As a result, it's been achieved an own conception of transmedia which allow us to define and to set the phases that this kind of storytelling has gone through on Harry Potter: from the boom of the early years and the later stagnation, to the new era that has just begun with the release of the new serie of films, that promises to reiterate the success of the previous years.

Key Words: Transmedia - Storytelling - Digital Communication - Platforms - Harry Potter - Adaptations - Consumers 


\section{Introducción}

La aparición del transmedia ha abierto una nueva puerta a la comunicación en red. Con el nacimiento de este tipo de narrativas han surgido otras formas de narrar historias y transmitirlas a una sociedad digitalizada cada vez más exigente con qué recibe y con cómo lo recibe. El concepto de narrativas transmedia fue originalmente introducido bajo el nombre de "intertextualidad transmedia" por Marsha Kinder en Playing with power in movies, television and video games (Kinder, 1991). No obstante, fue el académico Henry Jenkins quien años después las bautizó con el nombre que tienen hoy en día y las definió por primera vez en su artículo Transmedia Storytelling. Moving characters from books to films to video games can make them stronger and more compelling (Jenkins, 2003).

En los inicios del concepto, Jenkins definió las narrativas transmedia como:

(...) un proceso donde los elementos de una ficción se dispersan a través de múltiples plataformas mediáticas con el propósito de crear una experiencia de entretenimiento coordinada y unificada (...). En la forma ideal de narrativa transmedia, cada medio hace lo que se le da mejor, haciendo una contribución única y valiosa al desarrollo de la historia. De este modo, un relato puede ser introducido en un largometraje, expandirse en la televisión, novelas y cómics, y ese mundo puede ser explorado y vivido a través de un videojuego. Cada franquicia debe ser lo suficientemente autónoma para permitir un consumo independiente. 0 sea, no es necesario ver la película para entender el videojuego y viceversa (Jenkins, 2003).

A la hora de plantear un producto como transmediático, autores como del CE0 de Starlight Runner Entertainment y productor transmedia Jeff Gomez defienden, no sin razón, la necesidad de establecer una planificación previa de ese universo transmedia. Es decir, abogan por la proliferación de los universos nativos transmedia y critican las dificultades de expansión que presentan los no nativos. En palabras de Gomez: 
4 | María Melle Goyanes, Alicia Pardo Crego, Carlos Toural Bran y Xosé López García ARTÍCULOS DE INVESTIGACIÓN

Si no hay una estrategia de narrativas transmedia, entonces debes sentarte, esperar que tu contenido tenga éxito, y empezar a considerar de nuevo cuál va a ser tu próxima historia (...) La planificación transmedia ayuda a desarrollar una narración que nos permite ver la dirección de esas nuevas historias. Estamos preparados desde el principio para desarrollar contenido que se sume a un mundo en curso, en lugar de que los escritores y productores tengan que adivinar qué hacer en el próximo capítulo (Melle, Pardo, 2016).

Si se habla de los ejemplos de proyectos transmediales de ficción más relevantes de los últimos años, es necesario mencionar Pokémon y el Universo Cinematográfico de Marvel como universos transmedia nativos, y Lost, Star Trek y Star Wars como no nativos. Sin embargo, si nos fijamos en uno de los que está teniendo una expansión mayor y más variada en la actualidad, es inevitable hablar del caso del no nativo Harry Potter.

Tras los libros, las películas, los videojuegos, el merchandising y los parques temáticos, la gallina de los huevos de oro sigue trabajando. En noviembre de 2016 se estrenó un nuevo filme que trata sobre el Mundo Mágico a principios del siglo XX (la primera parte de la saga de Animales Fantásticos y Dónde Encontrarlos); el pasado verano fue presentada en Londres la obra de teatro sobre el hijo de Harry Potter (Harry Potter and the Cursed Child), así como el libreto en formato físico y digital y, a principios de este año 2017 salió publicado el guión de la película sobre Animales Fantásticos.

Para poder sacar adelante este tipo de producciones, por supuesto, es importante tener una buena idea, y disponer de los medios necesarios, también; no obstante, es necesario además valorar el papel crucial que desempeñan los fans, los receptores de estos productos, en cualquier universo transmedia. Muchos de ellos van un paso más allá de la figura del consumidor pasivo, y se convierten en creadores de contenido, en prosumidores: páginas de noticias, fanfics, fan arts, cortos, musicales, y un largo etcétera que contribuye a engrosar las dimensiones de este y otros universos transmedia. No hay duda de que, sin el interés del público, historias como las de Harry Potter jamás podrían haber alcanzado una dimensión semejante a la que tienen hoy en día. 
Es precisamente el gran número de extensiones que sigue generando en la actualidad lo que convierte al mundo creado por J.K. Rowling en uno de los mejores ejemplos transmediales de los últimos años. Sin embargo, a la hora de analizarlo, surgen una serie de cuestiones relativas a qué entra dentro de este concepto y qué se queda fuera. ¿Expanden las adaptaciones esta clase de universos? ¿Y las críticas y las reseñas? ¿Existe un mínimo de extensiones para considerar un producto como transmedia?

Y es que, aunque la idea general parece estar clara, las opiniones difieren a la hora de responder a estas preguntas, especialmente en lo relativo a las adaptaciones.

La australiana Christy Dena (2009) fue la primera en defender extensamente en su tesis doctoral por qué las adaptaciones deberían considerarse como parte de un universo transmedia:

(...) las adaptaciones no siempre son (o nunca son) redundantes. (...) el fenómeno del transmedia no se entiende mejor gracias a rasgos de productos finales como las expansiones, sino gracias al conocimiento y a las habilidades necesarias para crear (y experimentar) un proyecto transmedia. Estos conocimientos y habilidades implican el empleo de una variedad de medios (y entornos) distintos, de distintas maneras. Una de esas maneras es la adaptación.

Con el tiempo, también Jenkins llegó a aceptar la posibilidad de que parte de estos productos formasen parte del universo narrativo, y en la actualidad habla de que:

Ha habido una tendencia a distinguir entre extensiones transmedia y adaptaciones en la base de este concepto de comprensión añadida. Es decir, una extensión es conscientemente añadida al mundo ya existente, pretende alterar el modo en que lo entendemos. Una adaptación, por otro lado, vuelve a presentar la ficción, contándola de nuevo, para una nueva audiencia y a menudo hace mínimas adiciones al material original. Hay, obviamente, una línea difusa entre estos dos conceptos, especialmente desde que la mayoría de las adaptaciones aportan nuevas interpretaciones al material (Melle, Pardo, 2016). 
Por lo tanto, el autor deja la puerta abierta a la consideración de las adaptaciones como transmedia en función de lo que se crea que esa adaptación aporta. Lo mismo opina el productor transmedia Jeff Gomez (Melle, Pardo, 2016), quien asegura que solo las aceptaría como transmedia en el caso de que añadiesen nuevo contenido que hiciese que la historia se entendiese o se disfrutase mejor.

Sobre el mismo tema, Scolari también es ambiguo, aunque deja entrever que está a favor de considerar las adaptaciones como transmedia. Esto se puede ver a través de algunas de las declaraciones que vuelca en su libro Narrativas Transmedia: Cuando todos los medios cuentan, en el que por ejemplo, asegura, que "todas las adaptaciones presentan reflejos diversos, incluyen algún aroma nuevo y dejan algún retrogusto diferente al original" (Scolari, 2013, p. 49).

Por último, la investigadora en ciencia ficción y transmedia, María del Mar Grandío, se muestra mucho más clara, definiendo las adaptaciones como parte del universo transmedia de una historia sin ningún tipo de condicionante:

Estarían dentro de las narrativas transmedia como una parte complementaria y al mismo tiempo autónoma, porque en una adaptación, aunque evidentemente va a haber una relación muy directa con una narrativa central, siempre hay una interpretación y una transposición de ese contenido, y por lo tanto no es idéntica al original (Melle, Pardo, 2016).

Otro de los temas discutidos en este campo es si se deberían considerar las reseñas y derivados como parte del universo transmedia de un producto. En cuanto a este tipo de extensiones, Jenkins (Melle, Pardo, 2016) no se posiciona claramente, alegando que si bien es cierto que esta clase de contenido puede influir en cómo determinados grupos de consumidores entienden un mundo narrativo, también cree que pueden no añadir nada por sí mismos e incluso llegar a ser hostiles con el original.

Estas son solo algunas de las dificultades con las que el transmedia está lidiando en la actualidad. Otras, en cambio, se cerraron hace tiempo. Gomez (Scolari, 2013, p. 43), por ejemplo, defendía que el contenido se debía distribuir en tres o más 
La dimensión transmedia de Harry Potter: rasgos de las extensiones canónicas | 7

ARTÍCULOS DE INVESTIGACIÓN

plataformas de medios para poder considerarse transmedia. Hoy en día, ya no lo ve como un requisito imprescindible:

En los comienzos de las narrativas transmedia (...) sentí que era importante hacer que esta técnica fuera un poco más compleja para que los ejecutivos entendiesen el valor y la inversión necesaria para crear buenas narrativas transmedia, y no trozos de publicidad para fans. Las tres plataformas no son más que un requerimiento, ahora que entendemos mejor el transmedia (Melle, Pardo, 2016).

No obstante, e independientemente del origen de la narrativa o del número de extensiones que se generen a su alrededor, parece importante destacar que, como en cualquier historia, es fundamental tener un argumento con la calidad suficiente como para despertar el interés del público. En el caso estudiado para esta investigación, parece evidente que lo han conseguido.

\section{Metodología}

Esta investigación ${ }^{1}$ forma parte de un estudio previo del universo de Harry Potter que tiene como objetivo principal el análisis individual de sus distintas extensiones, así como la reelaboración de la definición de narrativas transmedia en base a las teorías de los académicos, y a partir de la revisión de sus publicaciones y de las entrevistas realizadas para este estudio. Como objetivos específicos, se plantea la clasificación del transmedia en el universo de Harry Potter, para poder analizar cómo funcionan estos tipos de narrativas en un caso práctico, al tiempo que se recoge la evolución de sus extensiones desde sus inicios hasta la actuali$\mathrm{dad}^{2}$. También se propone plasmar de forma gráfica las distintas plataformas del Mundo Mágico de Harry Potter en dos mapas y una cronología que muestren la magnitud del universo de Rowling, así como reunir todo el material analizado sobre este producto en una web propia que sirva de utilidad tanto a teóricos como a fans de la saga. 
Para la realización del proyecto y a fin de alcanzar los objetivos, tras una revisión bibliográfica, se han empleado métodos cuantitativos, mediante la técnica del análisis de contenido, para estudiar el caso particular de Harry Potter. A partir de los resultados de dicho análisis, se han formulado entrevistas estructuradas a fans y a expertos, de las que se ha extraído nuevo material destinado, primero, a la actualización de las informaciones del marco teórico $\mathrm{y}$, segundo, a la obtención de más datos sobre el producto con los que completar el análisis interpretativo de sus características y nuestras conclusiones al respecto.

De este modo, el desarrollo del trabajo se ha dividido en un total de 4 fases. Para estudiar la parte teórica del concepto central se ha atendido, por una parte, a la bibliografía que los investigadores de referencia consideran básica, de donde se han extraído la idea general y las teorías de los principales expertos sobre este campo; $\mathrm{y}$, por otra, a la bibliografía complementaria que a las autoras de la investigación les ha parecido que contenía datos particularmente significativos para delimitar el trabajo de acuerdo con los objetivos planteados; datos que han sido posteriormente completados gracias a las entrevistas ya mencionadas.

Así pues, en segundo lugar se han formulado una serie de cuestiones relacionadas con este tema a diversas fuentes. En el campo de los expertos, se ha hablado con Henry Jenkins, gurú indiscutible en lo que a teoría transmediática se refiere; Jeff Gomez, CE0 de Starlight Runner Entertainment, una productora centrada en la realización de proyectos transmedia como La Fábrica de la Felicidad de Coca-Cola; María del Mar Grandío, profesora del Departamento de Comunicación de la Universidad de Murcia que ha realizado diversas investigaciones centradas en la ciencia ficción en el transmedia; y Fernando Carrión, productor creativo transmedia y miembro del Transmedia Living Lab.

Para entender mejor el funcionamiento interno de este universo en concreto, se ha entrevistado a Eva Congil y Rafael Luna, dos de los responsables de la editorial Ediciones Salamandra, encargada de la publicación de todos los libros relacionados con Potter en España; a Neil Blair, el agente literario de J.K. Rowling que es, además, uno de los productores ejecutivos de la nueva saga de películas 
sobre Animales Fantásticos y director de Pottermore; y a varias figuras destacadas del fandom que han contribuido a expandir el universo a través de múltiples plataformas, como Pablo C. Reyna, fundador de la web hispana HarryLatino; Heather Lawver, creadora de la web The Daily Prophet y una de las cabecillas de las Potter Wars a principios de los años 2000; o Steve Vander Ark, autor del libro The Lexicon: An Unauthorized Guide to Harry Potter Fiction and Related Materials, denunciado por Rowling por plagio.

La tercera fase se ha basado en la propuesta de Jennifer García Carrizo y Olga Heredero Díaz (2015), a partir de la que se ha creado una ficha de análisis propia para el análisis de las extensiones. Consta de cuatro partes:

- Identificación y análisis de la macrohistoria o narrativa central: explicación del origen del universo Potter y el medio en el que nace, sus audiencias, las partes en las que se divide la narración y los aspectos narrativos (tiempo, espacio y escenarios en los que se desarrolla; así como los personajes clasificados según sus roles). A estos puntos se añade, además, una breve descripción de la trama.

- Identificación y análisis de las extensiones de la narrativa central que conforman la narración transmedia (tanto oficiales como extraoficiales): indicación del soporte en el que se desarrolla, sus audiencias (en función de la participación y del consumo de las mismas), las partes en que se divide y sus aspectos narrativos atendiendo a la intertextualidad del relato (es decir, si esas extensiones expanden el mundo ficcional, profundizan en personajes $\mathrm{u}$ otros elementos, o completan la historia incompleta). En el caso de las audiencias, se han unificado dos propuestas: la de García y Heredero sobre la participación, y la de Scolari sobre el consumo, al considerar que ambos puntos de vista aportan una visión mucho más completa del papel de los usuarios en estas extensiones. Sin embargo, en la clasificación de Scolari se omite la figura del consumidor de un único texto (aquel que solo consume lo relativo a la narrativa central, es decir, el producto principal en torno al que se genera el resto del universo), porque esa categoría solo estaría presente en el estudio de la misma. A estos puntos del análisis, se propone otro más: 
una introducción sobre cada plataforma para que el lector, aún sin conocerla, pueda hacerse una idea de cómo funciona, cuán compleja es y qué tipo de contenido añade.

- Elaboración de dos mapas sobre el universo transmedia oficial y extraoficial o fan (uno sobre el mundo de Harry Potter y otro sobre el subuniverso que se está generando alrededor de Animales Fantásticos), con el fin de reunir todos los elementos que conforman el mundo transmedia de Harry Potter de una forma visual.

- Elaboración de una línea cronológica de la evolución del transmedia en Harry Potter desde 1997 hasta la actualidad.

Finalmente, y como parte práctica, se han agrupado todas las plataformas estudiadas en una web de creación propia: El Traslador ${ }^{3}$. Debido a la limitación de espacio, no ha sido posible volcar aquí ni el análisis completo de cada una de las extensiones, ni las entrevistas anteriormente mencionadas. Así pues, se recomienda encarecidamente la consulta de esta web, en donde se recogen tanto los soportes analizados e indexados, como otros similares que puedan resultar de interés, además de todas las entrevistas, transcritas o en vídeo, realizadas a lo largo del proyecto.

\section{Una aproximación conceptual y de procedimiento}

Como fase previa al análisis, se consideró necesario establecer una definición propia que ayudase a delimitar los márgenes del universo transmedia que se iba a estudiar. Tras la revisión de las distintas teorías y autores, se ha llegado a la conclusión de que las narrativas transmedia pueden considerarse como el arte de expandir una idea inicial (la narrativa central) a través de múltiples plataformas de manera intencionada si es nativo (se concibe como transmedia desde el inicio) o no (se vuelve transmedia a raíz de su éxito). Cada una de estas extensiones es complementaria de la idea inicial y puede ser llevada a cabo tanto por fans como por los propios autores de la idea, pero siempre debe añadir algo nuevo que contribuya a ampliar el mundo narrativo. 
La dimensión transmedia de Harry Potter: rasgos de las extensiones canónicas | 11

ARTÍCULOS DE INVESTIGACIÓN

Este planteamiento coincide con los de Dena y Grandío al aceptar en cualquier caso las adaptaciones como parte del mundo transmedia de una historia, ya que siempre incluyen contenido de algún modo novedoso para el universo narrativo. Las reseñas, las críticas y los ensayos; así como las adaptaciones al braille, los audiolibros y las traducciones, por su parte, no entrarían de ninguna forma dentro de esta concepción de transmedia. Este material no contribuye a expandir el universo $\mathrm{y}$, de hecho, en lo que respecta a las críticas, en el caso de no ser constructivas, el objetivo no es en ningún caso crear, sino más bien destruir.

De este modo, y concretamente en el caso de Harry Potter, se incluyen las películas en la lista de plataformas a estudiar como parte de su universo narrativo ya que ofrecen una nueva perspectiva. Así mismo, se consideran como transmedia las wikis y las bandas sonoras de las extensiones (también denominadas "subproductos"). En el caso de las wikis, añaden nuevos datos (como por ejemplo el origen de algún elemento de la saga); y en lo que respecta a las bandas sonoras, se pueden consumir por separado y ayudan al espectador a profundizar en la acción.

Sin embargo, las críticas como Harry Potter Witchcraft Repackaged (Jeremiah Films, 2001), quedarán excluidas por ser, como dice Jenkins, hostiles con el original y no añadir nada por sí mismas a la narración (Melle, Pardo, 2016).

Teniendo esto en cuenta, se ha llevado a cabo un análisis de la narrativa central y de todas las plataformas a través de las cuales se expande la saga de Harry Potter. Atendiendo al medio en el que se presentan, a sus audiencias, sus partes y sus aspectos narrativos, en la clasificación de las extensiones se parte de dos diferencias básicas: plataformas oficiales (reconocidas y/o firmadas bajo el sello de J.K. Rowling) y plataformas extraoficiales o fans (las creadas generalmente por los seguidores de la historia). En el caso de estas últimas, ante la imposibilidad de identificar todas y cada una de las existentes debido al gran número de ellas (solo los relatos de los fans se cuentan por cientos de miles), se ha hecho un análisis más superficial, seleccionando todos los tipos de medios a través de los que se expanden y estudiando sus características en base a algunos de los mejores ejemplos de cada soporte, es decir, aquellos con un mayor número de usuarios y/o visitas 


\section{ARTÍCULOS DE INVESTIGACIÓN}

(HarryLatino.com, Potter Puppet Pals: The Mysterious Ticking Noise), los más polémicos (La Guía Secreta de Harry Potter, Magic Beyond the Words) o los más peculiares (torneos de Quidditch muggle). Como resulta evidente, este sesgo hace que el estudio se centre fundamentalmente en las extensiones del canon y obliga a dejar fuera interesantes iniciativas fans tales como el libro The Lexicon, la webserie Hermione Granger and the Quarter of Life Crisis o la organización sin ánimo de lucro The Harry Potter Alliance las cuales, sin duda, sería interesante abordar en un futuro estudio complementario.

\section{Resultados del análisis}

El análisis realizado muestra que el universo canon de Harry Potter ha pasado por un total de cuatro fases muy diferenciadas. Las dos primeras, centradas fundamentalmente en expandir la narrativa central; las otras dos, enfocadas en un proyecto mayor: asegurarse de que el mundo imaginado por Rowling no terminase tras haber agotado las plataformas relacionadas con la idea original. Tras un estudio más riguroso, se ha puesto de manifiesto que los medios más empleados a la hora de ampliar este universo han sido fundamentalmente los libros, que la función mayoritaria es la de profundizar en los elementos del relato, y que la mayoría de las extensiones se dirigen sobre todo a usuarios observadores (consumidores que no dejan una marca en los productos que consumen, pero que sí afectan a datos relacionados con ellos, como en el número de visitas o en la cantidad de ventas registradas).

Todos estos resultados se reflejan en mayor profundidad en la página web de El Traslador, y se plasman de manera gráfica en los mapas y la cronología que se presentan a continuación.

En el caso del primero de ellos (Figura 1), se representan de forma visual todas las extensiones que se han generado alrededor de la macrohistoria o narrativa central de Harry Potter (destacada en rojo). 
La dimensión transmedia de Harry Potter: rasgos de las extensiones canónicas | 13 ARTíCULOS DE INVESTIGACIÓN

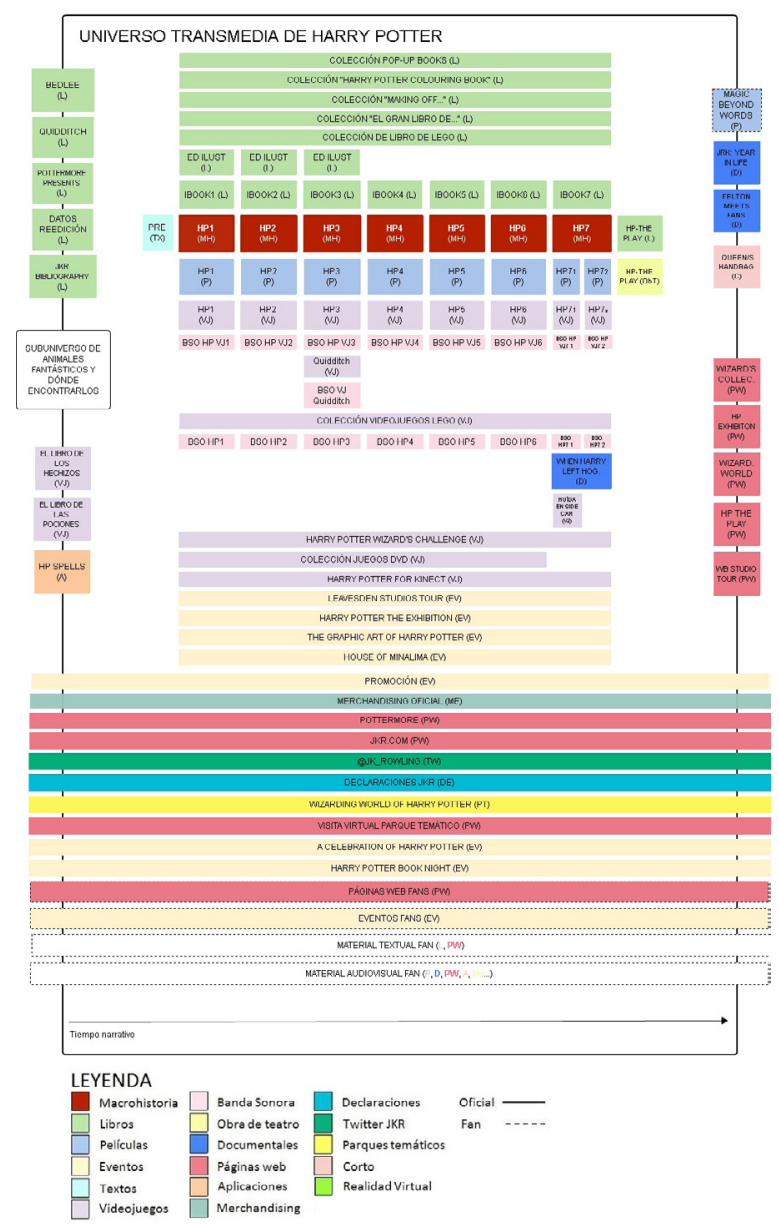

Figura 1: mapa del universo transmedia de Harry Potter. Fuente: creación propia

En el segundo (Figura 2) se muestra el subuniverso que se ha empezado a crear alrededor de Animales Fantásticos. En este caso, la narrativa central sigue siendo un libro, el de Animales Fantásticos y Dónde Encontrarlos, por ser el relato original. No obstante, en este mundo es necesario indicar una peculiaridad: pese a que el precedente es el texto escrito por Rowling, el universo se está desarrollando casi por completo alrededor de una de sus extensiones, la saga de películas. Y es que, al contrario de lo que ocurrió con las cintas de Harry Potter, los nuevos filmes no podrán ser considerados bajo ningún concepto como adaptaciones del relato que Rowling publicó en 2001, ya que apenas guardan relación con él. Sin embargo, esta distancia en los argumentos no 
convierte a las producciones cinematográficas en historias periféricas, sino en precuelas ya que, mientras que el libro simula ser un manual sobre criaturas mágicas escrito en 1927 por un mago, Newt Scamander; las películas se ambientan unos años antes y narran las aventuras que vivió el autor ficticio antes de la publicación de dicho libro.

Dejando a un lado estas diferencias, en ambos mapas cada tipo de soporte está identificado con un color propio (indicado en la leyenda) $\mathrm{y}$, para diferenciar entre productos oficiales y fans, que se recogen simbólicamente en las tres figuras, estos últimos están enmarcados por una línea discontinua. Su colocación se corresponde con el desarrollo del tiempo narrativo respecto a la narrativa central (de izquierda a derecha). Finalmente, aquellas extensiones que guardan tan solo una ligera relación con la trama central (periféricas) se sitúan a los márgenes, en los límites del universo transmedia.

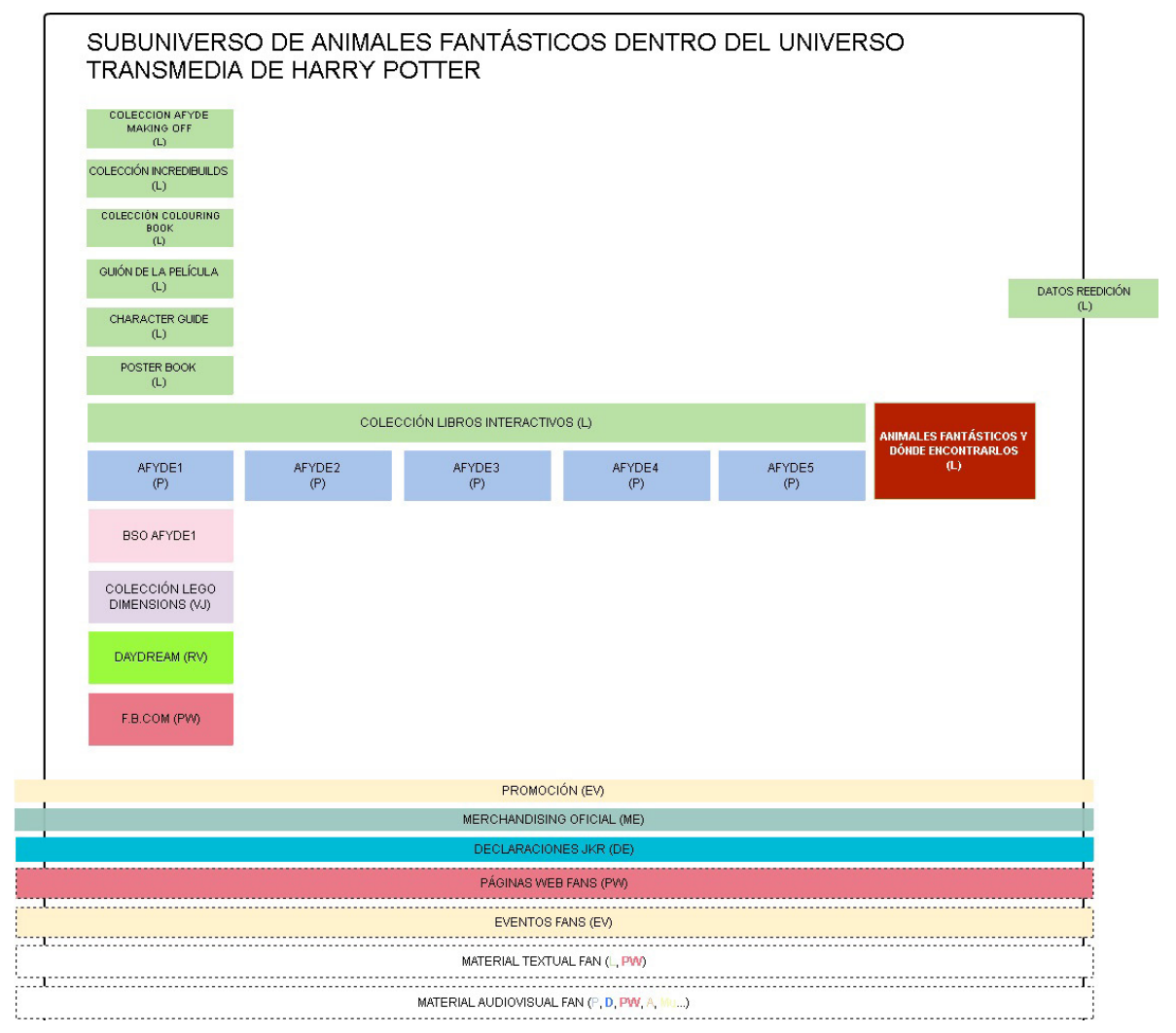

Figura 2: mapa del subuniverso transmedia de Animales Fantásticos. Fuente: elaboración propia. 
La dimensión transmedia de Harry Potter: rasgos de las extensiones canónicas | 15 ARTíCULOS DE INVESTIGACIÓN

La cronología (Figura 3), por su parte, presenta las extensiones dentro de una línea temporal y no narrativa, mostrando en qué años se produce una mayor afluencia de nuevas plataformas.

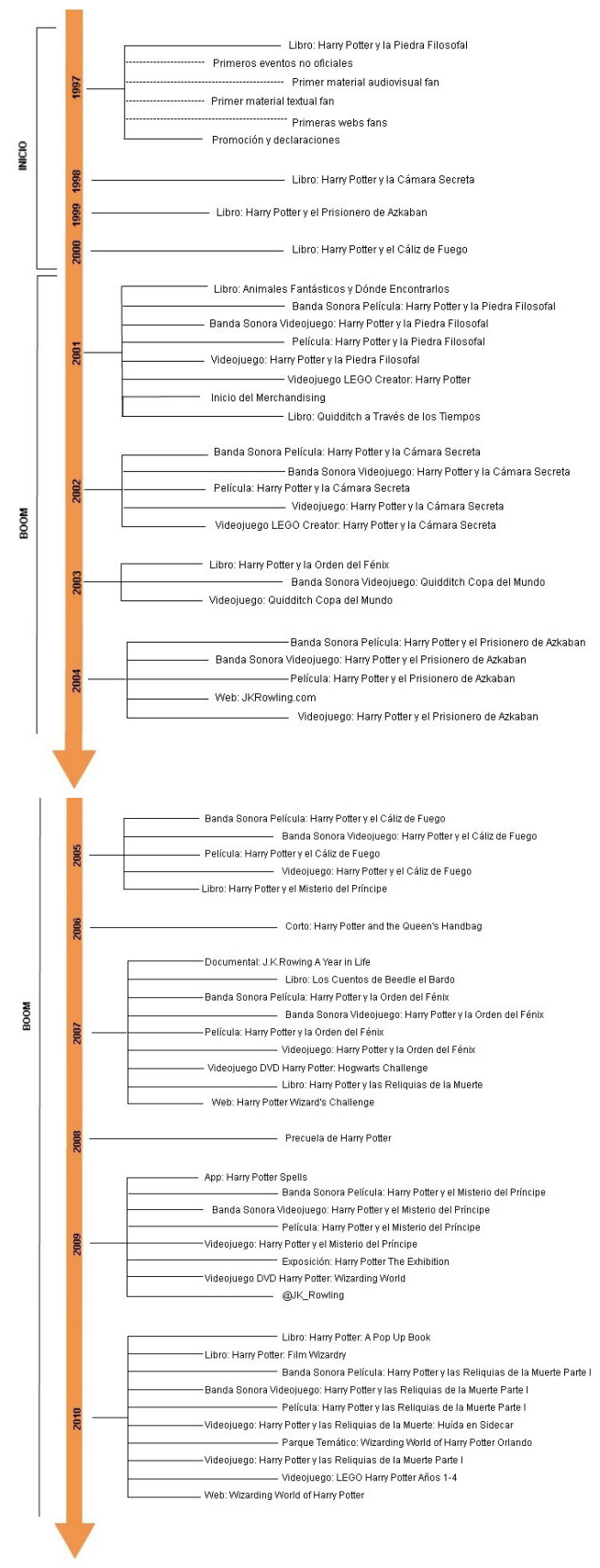

DOI: ri14.v15i2.1023 | ISSN: 1697-8293 | Julio - diciembre 2017 Volumen 15 N$^{\circ} 2$ | ICONO14 
16 | María Melle Goyanes, Alicia Pardo Crego, Carlos Toural Bran y Xosé López García ARTÍCULOS DE INVESTIGACIÓN

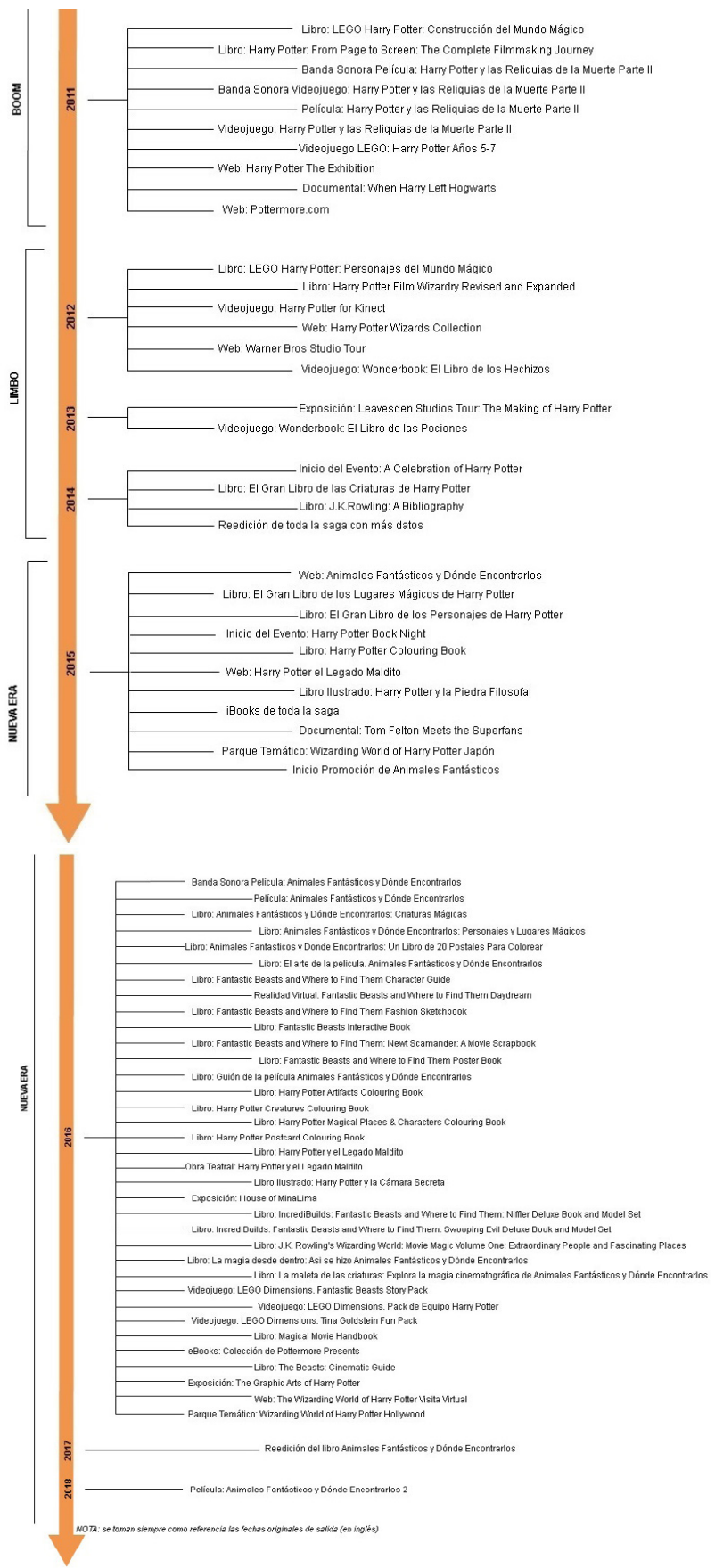

Figura 3: cronología de la evolución del universo transmedia de Harry Potter. Fuente: elaboración propia. 
La dimensión transmedia de Harry Potter: rasgos de las extensiones canónicas | 17

ARTÍCULOS DE INVESTIGACIÓN

Atendiendo a esta última figura, se pueden apreciar una serie de fases muy diferenciadas en la evolución del universo de Harry Potter. La primera, de 1997 al 2000, denominada "Inicio", engloba los comienzos de este mundo, en el que las únicas extensiones surgidas eran las creadas por los fans. En paralelo a estas plataformas, se publican en estos años las cuatro primeras partes de la narrativa central. Entre el 2001 y el 2011, tiene lugar el denominado "Boom". Durante esta década se lanzan, además de los tres libros restantes del relato central, las ocho películas de la saga de Harry Potter, que generan un mayor movimiento del fandom y provocan que la historia llegue a un público mucho más amplio. En este periodo se presentan cerca de un centenar de nuevas extensiones canónicas, la mayoría de ellas comerciales, relacionadas con las producciones cinematográficas y con poco valor añadido: videojuegos, bandas sonoras, libros basados en los filmes, documentales, un cortometraje,... Aún así, en esta etapa también surgen unas cuantas plataformas especialmente relevantes para la expansión del mundo narrativo de Potter, como la web de Pottermore, el primer parque de atracciones del Wizarding World, los tres libros de La Biblioteca de Hogwarts y la Precuela de Harry Potter.

A partir del 2012, y hasta el 2014, con los libros y las películas de la narrativa central ya acabados, el universo entra en un "Limbo" en el que Warner Bros. intenta mantener el engagement con el universo de Harry Potter lanzando una serie de extensiones bastante superfluas y sin mayor relevancia. Estos años están dominados sobre todo por las reediciones, los eventos vinculados a las películas y algún que otro videojuego. Aunque es cierto que este fue un momento en el que no hubo grandes aportaciones al universo transmedia, sí que sirvió para que los fans sintiesen que Harry Potter era un producto que seguía en movimiento.

Y lo cierto es que, efectivamente, el fenómeno estaba lejos de terminar. En el 2015, comenzó la "Nueva Era" del Wizarding World, tal y como lo denominaron desde la propia productora. Por un lado, se anunció el estreno de la obra de teatro Harry Potter and The Cursed Child. Dicha obra empezó a representarse en Londres en julio de 2016 y, gracias al gran éxito cosechado entre el público y la crítica, el equipo encargado ya ha iniciado los preparativos para estrenar la función en Broadway en la primavera de 2018. Por otro lado, también se empieza a intuir la magnitud del nuevo universo que se está formando alrededor de lo que, al parecer, 


\section{ARTÍCULOS DE INVESTIGACIÓN}

será el futuro del mundo de los magos: los cinco filmes inspirados en el libro de Animales Fantásticos y Dónde Encontrarlos.

La salida a la luz de las extensiones de este subuniverso no significa, sin embargo, que se hayan abandonado por completo las extensiones directamente relacionadas con la narrativa central. Los dos últimos años han sido el momento de los libros para colorear, de las ediciones ilustradas, de los nuevos eventos y encuentros con los fans, de las exposiciones sobre los diseños presentes en las películas y de una nueva colección de relatos bajo el nombre de Pottermore Presents, que siguen la estela de La Biblioteca de Hogwarts. Esta coincidencia no es un hecho aislado. En este periodo se están siguiendo de manera intencionada los mismos pasos dados en su momento con Harry Potter: ya empiezan a aparecer los primeros libros sobre el rodaje, los videojuegos y las entrevistas con los actores y el equipo técnico, en donde se amplía información y se profundiza en temas que se pasan por encima en la cinta. De hecho, aún sin haberse estrenado siquiera la primera película, ya estaban anunciadas alrededor de una treintena de nuevas extensiones. $Y$, una vez más, el soporte predominante entre ellas vuelve a ser el libro.

Así pues, todo parece indicar que nos adentramos en un nuevo ciclo en el que se tratará de repetir el éxito de la saga anterior. Hasta el momento, solo podemos intuir todo lo que terminará por generarse alrededor de Harry Potter and the Cursed Child y, con el spin-off protagonizado por Newt Scamander, surgirá todo un nuevo universo narrativo que, aun estando conectado con la historia de Potter, podrá consumirse de forma independiente y creará a su alrededor su propio mundo multiplataforma.

Sin embargo, sin adelantarnos al futuro también pueden sacarse otra serie de conclusiones. Concretamente, atendiendo a las plataformas analizadas hasta el momento, se ve, por un lado, que el soporte más común dentro del universo de Harry Potter es el libro (Quidditch a Través de los Tiempos, Harry Potter y el Legado Maldito), seguido muy de cerca por los videojuegos (El Libro de las Pociones, LEGO Harry Potter Años 1-4) y las bandas sonoras (Harry Potter y el Prisionero de Azkaban, Harry Potter Quidditch Copa del Mundo). No obstante, y como resulta evidente, el medio con más afluencia de público es la película 
La dimensión transmedia de Harry Potter: rasgos de las extensiones canónicas | 19 ARTÍCULOS DE INVESTIGACIÓN

(Harry Potter y las Reliquias de la Muerte Parte 1, Animales Fantásticos y Dónde Encontrarlos).

En cuanto a las funciones, la que se repite prácticamente en todas las plataformas estudiadas es la de profundizar en los personajes u otros elementos de la saga (Los Cuentos de Beedle el Bardo, J.K. Rowling: A Year in Life). También son habituales aquellas extensiones que contribuyen a expandir el mundo ficcional, especialmente a través de historias paralelas (The Graphic Arts of Harry Potter) y periféricas (Harry Potter and the Queen's Handbag). En cambio, el número de ellas que completa la historia incompleta es casi nulo (Pottermore).

Respecto a la audiencia, si se presta atención a la participación, el tipo de usuario mayoritario es el observador (la Precuela de Harry Potter, Harry Potter The Exhibition), el discursivo (Harry Potter and Me, Harry Potter Magical Places \& Characters Colouring Book) y el creador de contenido que da a conocer el universo (The Wizarding World of Harry Potter, Warner Bros. Studio Tour). Por otro lado, en función del consumo, el público más numeroso es el transmedia (When Harry Lefts Hogwarts, J.K. Rowling: A Bibliography 1997-2013).

Estos resultados, como ya se ha dicho, se orientan únicamente a la parte oficial del mundo estudiado. El sector fan, con sus millones de extensiones, resultó inabarcable desde el principio y solo ha sido recogido de manera superficial en los mapas y la cronología. Estas plataformas, así como las oficiales, se tratan en mayor profundidad en la página web que ha sido desarrollada para albergar otra parte de este estudio: El Traslador, transmedia para muggles. La razón de su creación ha sido llevar este trabajo a dos perfiles de usuarios muy diferentes. Por un lado, los fans, a los que se cree que una página así les podría ser de utilidad para conocer otras caras del universo de Harry Potter. Y, por otro, a los académicos del transmedia, a quienes puede resultarles interesante de cara a futuras investigaciones sobre la saga. El Traslador quiere acercar el transmedia a los fans de Harry Potter, y Harry Potter a los académicos del transmedia. 


\section{Conclusiones y discusión de resultados}

El gran cambio que ha experimentado el universo transmedia de Harry Potter desde sus inicios hasta hoy es innegable: en este tiempo las extensiones han evolucionado de manera significativa y parece que tanto la autora como la productora comprenden cada vez mejor los requisitos que necesitan para seguir despertando el interés de su audiencia. El primer problema al que se enfrentó esta historia fue que Harry no nació transmedia, por lo que llevó mucho tiempo comprender que era un producto que seguía creciendo más allá de los libros y las películas.

Tras entender el filón económico de este tipo de narrativas, desde la directiva de Potter se potenció el nacimiento de nuevas extensiones que complementasen el mundo narrativo original. Si algo hay que reconocerle a Warner Bros. es su habilidad para mantener la atención de los usuarios aún después del final de la saga. Luego de la salida de la última película y sin perspectivas de nada nuevo en el horizonte, fueron capaces de seguir produciendo nuevas plataformas que, pese tener una calidad discutible, conseguían despertar el interés del público. Sin embargo, en ellas primaba más el seguir beneficiándose de un fenómeno a priori ya explotado, que el aportar valor añadido que pudiese enriquecer la historia. Harry Potter era en aquel momento, por lo tanto, un buen ejemplo de transmedia en cuanto a cantidad, pero no en cuanto a calidad.

No obstante, en los últimos tres o cuatro años, sí que ha habido un intento significativo por parte tanto de Rowling como de Warner de ofrecerle al público un contenido más rico: resulta evidente que la obra de teatro o la nueva película aportan información mucho más novedosa y compleja que la que pueden dar los libros sobre las películas o las páginas webs comerciales. Se intuye entonces la entrada de esta franquicia en una nueva etapa que se corresponde fundamentalmente con la nueva saga de Animales Fantásticos en la que, si bien se continúan apoyando en los productos con más tirón económico (las películas), y todavía se publican extensiones con muy poco valor añadido (los libros de colorear o el guion de Animales Fantásticos), también se empieza a apostar por plataformas en las que se vuelque contenido más útil para los usuarios: nuevas historias sobre el Mundo Mágico en Pottermore, datos innovadores en eventos como A Celebration of Harry Potter, el videojuego de realidad virtual para Daydream,... 
Tampoco los fans parecen tener intención de dejar de crear su propio contenido, tengan o no el consentimiento de Warner y Rowling. Eventos como la primera PotterCon de Barcelona o la novena edición del Campus Potter vieron la luz este verano, fan films como el de Teddy Lupin y el Último Giratiempo siguen llamando la atención de muchos de los seguidores de Potter, webs como la de Blog Hogwarts todavía cuentan sus visitas diarias por varias decenas de miles,... Cientos y cientos de creaciones fans en todo el mundo que, lo quiera Rowling o no, siguen contribuyendo de manera innegable a engrosar el mundo de Harry Potter.

Lo cierto es que desde un principio la autora se mostró muy reacia a dejar que terceras personas creasen contenido a partir de sus historias, por lo que marcó unos límites muy claros para su universo, tratando de crear una narrativa totalmente cerrada. No obstante, a medida que el fenómeno ha seguido creciendo, J.K. ha tenido que ceder ante la gran cantidad de nuevas y variadas plataformas que surgían irremediablemente alrededor de su relato. De hecho, ahora es habitual que comparta a través de su cuenta personal de Twitter las creaciones fans que más le gustan, como fanarts o cosplays. Así pues, de acuerdo con Neil Blair, en la actualidad los abogados de la autora solo toman medidas "(...) si hay algún tipo de infracción [de copyright] $\mathrm{y}$, en particular, si hay gente tratando de vender el libro o hacerlo parecer como si fuese escrito por Jo, entonces emprendemos acciones legales" (Melle, Pardo, 2016).

No obstante, esta ligera concesión no ha significado un gran cambio de actitud respecto a los prosumidores. Resulta sorprendente que después de tantos años y viendo lo involucrados que han estado siempre los fans, no haya todavía, salvo contadas excepciones, extensiones oficiales pensadas para promover su participación. Haciendo un repaso por todas las plataformas que forman el canon del mundo Potter se aprecia sin dificultades que la gran mayoría se dirigen únicamente a una audiencia pasiva. En un principio, este comportamiento era justificable, ya que, como se ha dicho, este universo no nació para ser transmedia, pero, a día de hoy, resulta inexplicable que ni la autora ni la productora hayan tratado de explotar más el papel del prosumidor. Sin embargo, y viendo el rumbo que parece que quieren empezar a tomar, tal vez en unos años podamos ver algunos avances significativos en este campo: la posibilidad de un concurso de fanfics o un festival de fanfilms no suena como algo tan descabellado, después de todo. 


\section{ARTÍCULOS DE INVESTIGACIÓN}

Lo que sí están sabiendo ya cómo explotar es la oportunidad ofrecida por una de las extensiones canon más compleja de su universo: Animales Fantásticos. A partir del libro, se creará una nueva saga de películas con la que volver a reiniciar el ciclo comenzado en el 2001 con Potter. La cuestión es, ¿por qué ahora? Como todas las minas, la de Harry ha terminado por agotarse. Llegados al punto en el que parece que este universo no se puede estirar mucho más, y aparcando (por el momento) la idea de un reboot, se ha decidido comenzar lo que desde Warner se denomina una "Nueva Era". Nuevos personajes, un nuevo argumento y un nuevo continente, pero, eso sí, la misma fórmula de siempre: el sello de Rowling y del Mundo Mágico de Harry Potter en todas y cada una de las extensiones lanzadas. Sin duda alguna, el éxito está asegurado.

\section{Notas}

[1] Este texto ha sido elaborado en el marco de los trabajos de investigación del grupo Novos Medios financiados con el Proyecto de I+D+i “Usos y preferencias informativas en el nuevo mapa de medios en España: Modelos de periodismo para dispositivos móviles" (referencia CS0201564662-C4-4-R) del Ministerio de Economía y Competitividad del Gobierno de España.

[2] El análisis abarca en concreto desde 1997 hasta octubre de 2016, momento en que fue enviado el documento. Todas las extensiones surgidas a posteriori no se recogen en esta investigación.

[3] Enlace a El Traslador: http://eltrasladortfg.wixsite.com/el-traslador

\section{Referencias}

Costa, C. (2013). Narrativas Transmedia Nativas: Ventajas, elementos de la planificación de un proyecto audiovisual transmedia y estudio de caso. Historia Y Comunicación Social, 18(0). Recuperado de https://goo.gl/jb4IiN

Costa, C., \& Piñeiro, T. (2012). Nuevas Narrativas Audiovisuales: Multiplataforma, crossmedia y transmedia. Revista ICON014. Revista Científica De Comunicación Y Tecnologías Emergentes, 10(2), 102. Recuperado de https://goo.gl/FwiQ0V

Dena, C. (2009). Transmedia Practice: Theorising the Practice of Expressing a Fictional World across Distinct Media and Environments. University of Sidney. Recuperado de https://dl.dropboxusercontent.com/u/30158/DENA_TransmediaPractice.pdf 
García Carrizo, J., \& Heredero Díaz, 0. (2015). Propuesta de un modelo genérico de análisis de la estructura de las narrativas transmedia / Proposal for a generic model of analysis of the structure of transmedia narratives. Icono 14, 13(2), 260. doi: 10.7195/ri14.v13i2.745

Jenkins, H. (2006). Convergence Culture. La cultura de la convergencia de los medios de comunicación. Barcelona: Paidós.

Jenkins, H. (2009). Revenge of the Origami Unicorn: The remaining four principles of transmedia storytelling. Confessions of an Aka-Fan. Recuperado de https:// goo.gl/DnCWlU

Jenkins, H. (2009). The Revenge of the Origami Unicorn: Seven Principles of Transmedia Storytelling (Well, Two Actually. Five More on Friday). Confessions of an Aka-Fan. Recuperado de https://goo.gl/nTYvUQ

Jenkins, H. (2011). Three Reasons Why Pottermore Matters.... Confessions of an Aka-Fan. Recuperado de https://goo.gl/BAXsqi

Jenkins, H. (2003). Transmedia Storytelling. Moving characters from books to films to video games can make them stronger and more compelling.MIT Technology Review. Recuperado de https://goo.gl/YJddbX

Kinder, M. (1991). Playing with power in movies, television, and video games. Berkeley, Calif.: University of California Press.

Melle, M., \& Pardo, A. (2016). Henry Jenkins - El Traslador. [online] El Traslador Transmedia para muggles. Recuperado de https://goo.gl/0ZBbJ0

Melle, M. \& Pardo, A. (2016). Jeff Gómez - El Traslador. [online] El Traslador Transmedia para muggles. Recuperado de https://goo.gl/MEA9pC

Olabarri, L. (2014). Narrativas transmedia: contar historias por todos los medios. CUV3. Recuperado de https://goo.gl/vSFtnH

Parada, N. (2015). Que teñen en común Matrix, Harry Poter e Lost?. Red Social del Grupo Stellae. Recuperado de https://goo.gl/FD4klw

Pottermore. (2015). Cursed Child is the 'eighth Potter story'. Pottermore. Recuperado de https://goo.gl/iSO2II

Pottermore. (2016a). Artist Jim Kay on illustrating Harry Potter. Pottermore. Recuperado de https://goo.gl/ZYKU4v

Pottermore. (2016b). Exciting publishing programme from J.K. Rowling's Wizarding World. Pottermore. Recuperado de https://goo.gl/2aLrly 
24 | María Melle Goyanes, Alicia Pardo Crego, Carlos Toural Bran y Xosé López García

\section{ARTÍCULOS DE INVESTIGACIÓN}

Pottermore. (2016c). Fantastic Beasts and Where to Find Them screenplay to be published. Pottermore. Recuperado de https://goo.gl/Yq9HQ9

Rowling, J. (2009). J.K. Rowling (@jk_rowling). Twitter Recuperado de https:// goo.gl/V5tGVK

Rowling, J. (2016). History of Magic in North America. Pottermore. Recuperado de https://goo.gl/EJVhpd

Rowling. J. (2017). J.K. Rowling. Jkrowling.com. Recuperado de https://goo.gl/ vkPW8V

Scolari, C. (2012). Más allá de la ficción: el documental transmedia. Hipermediaciones. Recuperado de https://goo.gl/AJJrK5

Scolari, C. (2013). Narrativas transmedia. Cuando todos los medios cuentan. Barcelona: Deusto.

TEDx. (2010). TEDxTransmedia - Jeff Gomez - DAREtoChange. Recuperado de https:// youtu.be/p9SlVedmnw4 